\title{
Educar para el desarrollo sostenible en el contexto universitario: un análisis bibliométrico
}

\author{
María J. Alcalá del Olmo-Fernández¹, Carmen Rodríguez-Jiménez², María J. Santos-Villalba ${ }^{3}$, Gerardo Gómez-García² \\ (1) Universidad de Málaga, Facultad de Ciencias de la Educación, Dpto. de Didáctica y Organización Escolar, Málaga, \\ España. (correo-e: mjalcaladelolmo@uma.es) \\ (2) Universidad de Granada, Facultad de Ciencias de la Educación, Dpto. de Didáctica y Organización Escolar, \\ Granada, España. (correo-e: carmenrj@ugr.es; gomezgarcia@ugr.es) \\ (3) Universidad Internacional de La Rioja, Facultad de Ciencias de la Educación, Dpto. de Didáctica y Organización \\ Escolar. La Rioja, España. (correo-e: mariajesus.santos@unir.net)
}

Recibido Oct. 26, 2020; Aceptado Dic. 29, 2020; Versión final Ene. 22, 2021, Publicado Jun. 2021

\begin{abstract}
Resumen
El objetivo de esta investigación es analizar la productividad científica sobre la educación para el desarrollo sostenible en la educación superior. Los conflictos ecológicos constituyen uno de los fenómenos de mayor trascendencia para la humanidad. Todo ello repercute en los espacios educativos, reclamándose una educación comprometida con el respeto al planeta en todos los niveles formativos, incluido el universitario. Se emprende un estudio bibliométrico a partir de la revisión de 322 artículos científicos publicados desde el año 1970 al año 2019. Los resultados revelan una productividad creciente de investigaciones en los últimos años, lo que permite entrever la relevancia concedida a estas cuestiones en la comunidad científica. Se concluye que a pesar de que los índices de productividad de la educación para el desarrollo sostenible en el espacio universitario datan de varios años, la presencia de esta línea de investigación ha ganado relevancia en los últimos tiempos.
\end{abstract}

Palabras clave: desarrollo sostenible; educación superior; cambio social; innovación educativa; bibliometría

\section{Sustainable development education in the university context: a bibliometric analysis}

\begin{abstract}
The objective of this study is to analyze scientific productivity on the topic of sustainable development education in higher education. Ecological conflicts are among the most important phenomena for humanity. They have repercussions in educational spaces, demanding an education committed to teaching respect for the planet at all education levels, including at the university level. A bibliometric assessment is conducted based on the review of 322 scientific articles published from the years 1970 until 2019. The results reveal growing research productivity on sustainable development education in recent years, showing the relevance acquired by this topic in the scientific community. It is concluded that even though there is evidence of scientific productivity on sustainable development education for some time, research on this line of investigation gained relevance only recently.
\end{abstract}

Keywords: sustainable development; higher education; social change; innovation in education; bibliometrics 


\section{INTRODUCCIÓN}

En los últimos años, la convergencia de variables como el cambio climático, los conflictos bélicos en diversas partes del mundo y los movimientos migratorios han hecho de la sostenibilidad un desafío de gran magnitud para las actuales generaciones (Geli de Ciurana et al., 2019). Como consecuencia de los procesos de industrialización y la adopción de nuevos estilos de vida, se ha generado un incremento descontrolado de la explotación de los recursos naturales (Barata et al., 2017), emergiendo conflictos ambientales que reflejan las inadecuadas relaciones que el hombre establece con su entorno. Todo ello genera importantes implicaciones educativas, reclamando el valor de la educación como herramienta con la que impulsar el necesario cambio de valores, actitudes y procedimientos con los que respetar el Planeta. En este sentido, la Educación para el Desarrollo Sostenible emerge como una propuesta pedagógica inclusiva e innovadora que busca impulsar relaciones armónicas con el entorno, situando la mirada en un cambio de perspectiva, que lleve a la modificación de conductas en las formas de vivir, en las convicciones y en las prioridades (Álvarez-Lires et al., 2017).

A comienzos del siglo $\mathrm{XX}$, los organismos internacionales se encargaron de impulsar todo un conjunto de propuestas destinadas a la promoción de una educación respetuosa con el entorno, lo que reflejaría el compromiso internacional a favor de una Educación para el Desarrollo Sostenible (Amaral et al., 2015; Jorgenson et al., 2019). De este modo, en el horizonte temporal 2005-2014, la Asamblea General de las Naciones Unidas promulgó el Decenio de la Educación para el Desarrollo Sostenible, reclamando el valor de una educación de corte sostenible e inclusivo en todas las etapas educativas, incluida la universitaria, de forma que el paradigma de la sostenibilidad se hiciera palpable en aspectos relativos a la gestión, la docencia y la investigación, para ofrecer una formación superior de excelencia y comprometida con los conflictos socioambientales (Murga-Menoyo, 2018).

La Agenda 2030 para el Desarrollo Sostenible constituye el referente más ambicioso con el que hacer realidad los Objetivos del Desarrollo Sostenible aprobados por la Asamblea General de las Naciones Unidas para el espacio comprendido entre 2016-2030 (ONU, 2015), uniendo de forma indisoluble las actuaciones pedagógicas con los principios y ejes de sostenibilidad. Todo ello ha llevado a que el desarrollo sostenible repercuta en todos los campos de conocimiento, siendo el Espacio Europeo de Educación Superior la antesala para impulsar una educación con la que adquirir competencias destinadas a intervenir eficazmente ante los retos ecológicos que nos rodean desde la cotidianeidad y el ejercicio profesional (Unesco, 2017), acentuando la necesidad de apoyar la formación universitaria en un nuevo modelo, que permita el abordaje transversal de la sostenibilidad en todos los grados universitarios (Alcalá del Olmo, 2020; Alcalá del Olmo et al., 2020; Aleixo et al., 2018; Espino-Román et al., 2015).

De este modo, la universidad constituye un escenario privilegiado en el que diseñar propuestas pedagógicas con las que implementar una Educación para el Desarrollo Sostenible, teniendo en cuenta que el alumnado que se encuentra en formación ocupará en el futuro puestos profesionales en diferentes escenarios laborales, constituyendo un motor de cambio para responder a los nuevos desafíos ambientales. Desde esta perspectiva, la apuesta es integrar la sostenibilidad en los objetivos estratégicos de las universidades (Cebrián et al., 2020; Murga-Menoyo, 2018; Sjögren, 2019; Valderrama et al., 2020), lo que implica la adopción de cambios en la forma de concebir el currículum y los métodos tradicionales de enseñanza, incorporando los elementos centrales de la equidad y la justicia social en las diversas profesiones, para trabajar desde la responsabilidad y la ética hacia el desarrollo sostenible.

Durante las últimas décadas, ha sido creciente el interés de la comunidad científica en la revisión de las bases conceptuales de la Educación para el Desarrollo Sostenible, siendo profusas las investigaciones que centran su mirada en valorar los efectos de su implementación en el colectivo estudiantil y el profesorado de todas las etapas educativas, incluida la universitaria (Antúnez y Gomera, 2017; De Matos et al., 2020; Mulá et al., 2017; Purcell et al., 2019). De la misma forma, en la literatura científica se han encontrado publicaciones que analizan, desde una perspectiva bibliométrica, el número de investigaciones recientes relacionadas con esta disciplina en los contextos educativos (Medina y Páramo, 2014; Wright y Liang, 2019), si bien se ha podido observar que son escasos los estudios en los que se analice la producción científica de una educación vinculada con el paradigma de la sostenibilidad en el contexto universitario.

Es por ello por lo que se considera necesario realizar este estudio bibliométrico, cuyo objetivo es emprender un análisis de la producción científica existente relacionada con la puesta en práctica de una educación comprometida con la sostenibilidad en la Educación Superior. Dicho análisis puede ayudar a conocer e identificar el dinamismo y la trayectoria seguida por la Educación para el Desarrollo Sostenible, además del interés expresado por los profesionales de esta disciplina en la transmisión y transferencia de sus planteamientos a otros colectivos que compartan semejantes inquietudes en este ámbito del conocimiento. 


\section{MATERIALES Y MÉTODOS}

A lo largo de esta investigación se ha recurrido a una metodología propia de los estudios bibliométricos (Cruz, 1999; Fernández-Cano y Bueno-Sánchez, 1998), que permite analizar y evaluar la producción investigadora en un ámbito específico sobre una temática concreta (Diem y Wolter, 2013). La búsqueda y filtrado de la literatura científica empleada en el presente estudio tuvo lugar en el segundo trimestre del año 2020 en la base de datos Web of Science (WOS), al tratarse de un repositorio que goza de excelente rigor y prestigio en lo que respecta a los documentos científicos que alberga. Del mismo modo, ofrece la posibilidad de limitar y diseñar la búsqueda atendiendo a diferentes indicadores, lo que define su carácter concreto y personalizado.

Los metadatos que finalmente se obtuvieron fueron extraídos del análisis de otros estudios de corte semejante (Gutiérrez-Braojos et al., 2016; Wright y Liang, 2019), siguiéndose, como indicadores, el año de publicación, autores más prolíficos, trabajos más citados, instituciones con mayor producción científica, revistas más productivas y conexión entre descriptores.La confección de la muestra final $(n=322)$ se realizó tras la consecución de una serie de procesos de refinamiento, como se observa en la tabla 1. Los conceptos clave fueron buscados y delimitados en el Thesaurus ERIC. A partir de ello, pudieron extraerse las palabras clave "Education for Sustainable Development"; "Higher Education". Dichos términos fueron introducidos en la base de datos anteriormente citada con el booleano "AND", con el propósito de obtener literatura científica en cuyo título, resumen, palabras clave y cuerpo del texto estuviera presente la terminología clave objeto de estudio. Una vez delimitados los criterios de inclusión y exclusión, se procede a definir en la tabla 2 una delimitación de las diferentes preguntas, y, con ellas, los objetivos centrales que impulsan la realización de este trabajo.

Tabla 1. Criterios de inclusión y exclusión utilizados en el análisis bibliométrico.

\begin{tabular}{|l|l|}
\hline \multicolumn{1}{|c|}{ Criterios de inclusión } & \multicolumn{1}{c|}{ Criterios de exclusión } \\
\hline $\begin{array}{l}\text { Producción desde su origen al año 2019 } \\
\text { Artículos científicos }\end{array}$ & $\begin{array}{l}\text { Año 2020, por no estar acabado } \\
\text { Capítulos de libro, libro, editoriales, actas de congreso }\end{array}$ \\
\hline $\begin{array}{l}\text { Aplicación de la temática relacionada con } \\
\text { el medio ambiente en Educación Superior }\end{array}$ & Aplicación de la temática en otras etapas educativas \\
\hline
\end{tabular}

Tabla 2. Objetivos, preguntas de investigación e indicadores bibliométricos que dan respuesta.

\begin{tabular}{|c|c|c|}
\hline Objetivo & Pregunta & Indicador \\
\hline $\begin{array}{l}\text { Evolución del } \\
\text { área y su } \\
\text { productividad }(\mathrm{A})\end{array}$ & $\begin{array}{l}\text { ¿Cuándo fue el origen del tratamiento investigativo de la } \\
\text { Educación para el Desarrollo Sostenible en la educación? } \\
\text { ¿Cuál ha sido la evolución de esta disciplina en el marco } \\
\text { educativo? }\end{array}$ & $\begin{array}{l}\text { A.1. Número de artículos y } \\
\text { años de publicación. } \\
\text { A.2. Productividad } \\
\text { diacrónica y Ley de Price. }\end{array}$ \\
\hline $\begin{array}{l}\text { Características de } \\
\text { las revistas y } \\
\text { dispersión de la } \\
\text { producción } \\
\text { científica }(B)\end{array}$ & $\begin{array}{l}\text { ¿Cuáles son las principales revistas que publican sobre el impacto } \\
\text { de la Educación para el Desarrollo Sostenible en el escenario } \\
\text { universitario? } \\
\text { ¿Cómo se encuentran los artículos distribuidos en el total de } \\
\text { revistas productoras conformantes? }\end{array}$ & $\begin{array}{l}\text { B1. Revistas más } \\
\text { productoras sobre la } \\
\text { temática. } \\
\text { B2. Ley de Bradford. }\end{array}$ \\
\hline $\begin{array}{l}\text { Productividad del } \\
\text { autor e } \\
\text { instituciones más } \\
\text { prolíficas }(C)\end{array}$ & $\begin{array}{l}\text { ¿Cuáles son los autores más prolíficos? } \\
\text { ¿Cuál es el nivel de citación de los mismos y el índice de } \\
\text { impacto? } \\
\text { ¿Cuáles son las instituciones más productivas? } \\
\text { ¿Sigue el nivel de contribución de los autores un patrón } \\
\text { determinado? }\end{array}$ & $\begin{array}{l}\text { C1. Lista de autores más } \\
\text { productores sobre AC. } \\
\text { C2. Lista de las } \\
\text { instituciones más } \\
\text { prolíficas. } \\
\text { C3. Ley de Lotka. }\end{array}$ \\
\hline $\begin{array}{l}\text { Temáticas más } \\
\text { investigadas } \\
\text { dentro de la } \\
\text { muestra (D) }\end{array}$ & $\begin{array}{l}\text { ¿Qué temáticas son las más abordadas dentro de la Educación } \\
\text { para el Desarrollo Sostenible? } \\
\text { ¿Cuáles son las palabras clave y la conexión entre ellas en los } \\
\text { distintos artículos científicos? }\end{array}$ & $\begin{array}{l}\text { D1. Lista de tópicos más } \\
\text { tratados. } \\
\text { D2. Mapa de conexión } \\
\text { entre descriptores clave. }\end{array}$ \\
\hline
\end{tabular}

\section{RESULTADOS}

Se muestran seguidamente los resultados más significativos de este estudio, situando la mirada en la evolución y producción del área, los indicadores de dispersión y los indicadores de impacto.

\section{Evolución y producción del área}

Con respecto a la productividad diacrónica, cabe poner de relieve que, como resultado de la combinación de las palabras clave ya especificadas y la realización con las mismas de la búsqueda especializada, fueron obteniéndose resultados de diferente naturaleza comprendidos entre los años 1970 y 2019. Dicha productividad diacrónica queda especificada en la figura 1. En ella puede apreciarse que el inicio de la literatura científica tendría su origen en el año 1970, con una productividad de dos artículos científicos, alcanzando su máximo en el año 2018, con 44 trabajos. Tomando como referente la ley de Price (Price, 1986), 
se comprueba que la duplicidad científica no se produce hasta 10 años desde el surgimiento de la línea de investigación, sino que se comenzó a observar pasados hasta 30 años desde el primer artículo científico datado en Web of Science.

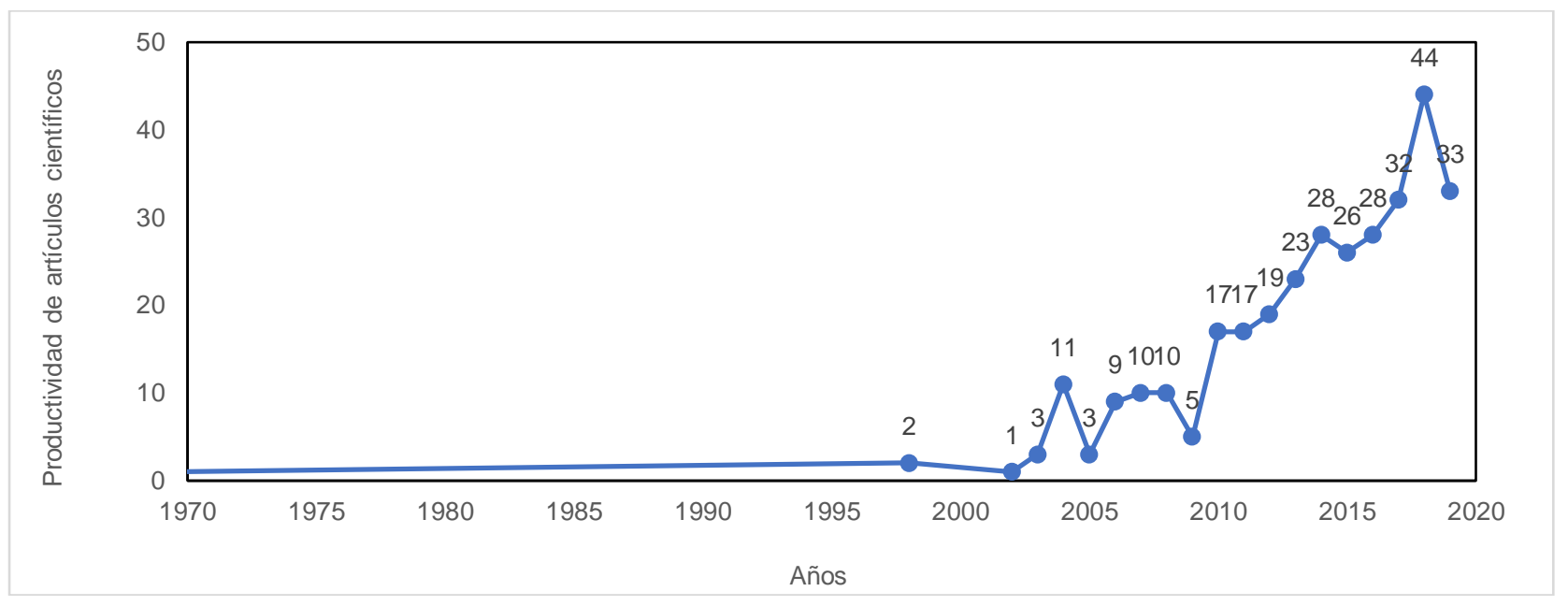

Fig. 1. Productividad diacrónica de la Educación Ambiental en la Educación Superior

\section{Indicadores de dispersión}

Siguiendo con los indicadores de impacto, la Ley Bibliométrica de Bradford, también conocida como Ley de Dispersión de la Literatura Científica, indica que un número reducido de revistas concentran un número de publicaciones que es igual o prácticamente similar a aquellas agrupadas en otros grupos de mayores revistas. Esta ley trata de demostrar que la distribución de la producción de artículos referentes a una temática es altamente desigual (Bradford, 1934; Miranda, 1990). Así, en la figura 2 se puede observar cómo las diferentes publicaciones quedan agrupadas en diferentes zonas (zona 1 y zona 2), las cuales, individualmente, engloban el mismo número o semejante de publicaciones que en el núcleo de la figura. El total consta de 171 revistas y publicaciones, distribuidas en tres zonas con una media de unas 100 publicaciones cada una, donde se corrobora que es el núcleo con tan solo 10 revistas en el que se encuentra un número de publicaciones (109) muy similar al resto de zonas.

\section{Indicadores de impacto}

Los indicadores de impacto han sido divididos en tres aspectos fundamentales, con el objetivo de conocer la influencia de la Educación para el Desarrollo Sostenible en el contexto universitario: revistas, instituciones y autores. De cada uno de ellos se han analizado las 10 revistas, instituciones o autores con mayor número de contribuciones. De este modo, en lo que respecta a las revistas más prolíficas en la tabla 3 se observa que destacan Revista electronica do mestrado em educacao ambiental (8.07\%), Environmental Educational Research (6.83\%), Sustainability (5.59\%) e International Journal of Sustainability in Higher Education (4.97\%). Por otro lado, como editoriales principales sobresalen Pereira, MDPI y especialmente, Taylor \& Francis.

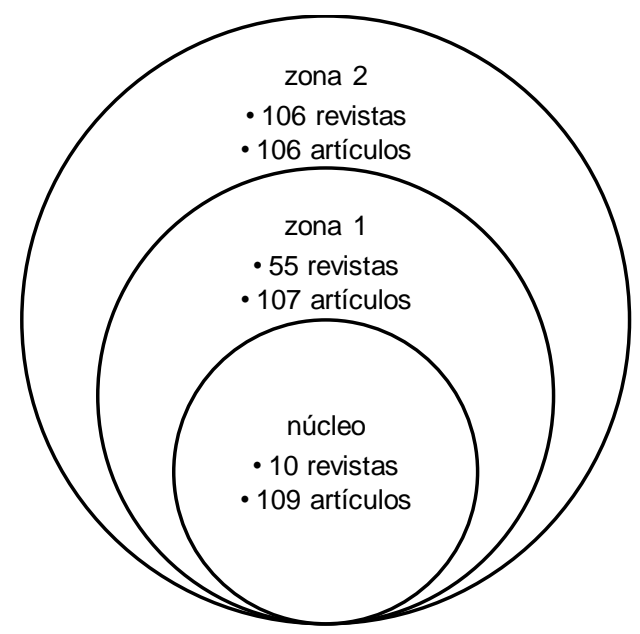

Fig. 2. Distribución de revistas y artículos en torno a las zonas de Bradford 
Tabla 3. Revistas con mayor número de artículos sobre Educación para el Desarrollo Sostenible en la Educación Superior

\begin{tabular}{|l|c|c|c|c|l|}
\hline Revista & No doc & $\%$ & Citas & Indice de impacto & \multicolumn{1}{|c|}{ Editorial } \\
\hline $\begin{array}{l}\text { Revista electronica do mestrado em } \\
\text { educacao ambiental }\end{array}$ & 26 & 8.07 & 26 & 1 & Pereira \\
\hline Environmental Educational Research & 22 & 6.83 & 282 & 12.81 & Taylor \& Francis \\
\hline Sustainability & 18 & 5.59 & 76 & 4.22 & Emerald Publishing \\
\hline $\begin{array}{l}\text { International Journal of Sustainability in } \\
\text { Higher Education }\end{array}$ & 16 & 4.97 & 195 & 12.18 & MDPI \\
\hline Journal of Cleaner Production & 11 & 3.41 & 426 & 38.72 & Elsevier \\
\hline $\begin{array}{l}\text { Revista Electrónica em Gestao Educacao e } \\
\text { Tecnologia ambiental }\end{array}$ & 8 & 2.48 & 5 & 0.625 & Santa Maria RS \\
\hline Water Sciences and Technology a Journal & 7 & 2.17 & 2 & 0.28 & IWA Publishing \\
\hline $\begin{array}{l}\text { Journal of Environmental Education } \\
\text { Australian Journal of Environmental }\end{array}$ & 5 & 1.55 & 30 & 6 & $\begin{array}{l}\text { American Society of } \\
\text { Education }\end{array}$ \\
\hline Journal of Geography in Higher Education & 4 & 1.24 & 54 & 13.5 & Cambridge Core \\
\hline
\end{tabular}

Si atendemos a los autores, la tabla 4 presenta los 10 autores más prolíficos sobre la temática. Se observa que la mayoría de autores presentan un total de 3 contribuciones. En el caso del índice de impacto de los autores, son Kophina, Shephard y Vega-Marcote aquellos cuyos trabajos presentan una mayor relevancia en la comunidad científica. Atendiendo a la ley de Lotka, se visualiza que no existe un cuerpo de expertos contrastado que se encargue de producir la mayor cantidad de la literatura científica sobre esta línea de investigación. Se observa que ninguno de ellos sobresale en número de artículos producidos, y que presentan una cantidad similar de artículos científicos.

Tabla 4. Autores con mayor número de contribuciones.

\begin{tabular}{|l|c|c|c|c|}
\hline \multicolumn{1}{|c|}{ Autor } & № doc & $\%$ & Citas & Índice de impacto \\
\hline Arevalo, J & 3 & 0.93 & 24 & 8 \\
\hline Berchin, L & 3 & 0.93 & 6 & 2 \\
\hline Comas-Forgas, R & 3 & 0.93 & 29 & 9.66 \\
\hline Correa, L.B. & 3 & 0.93 & 6 & 2 \\
\hline Kopnina, H. & 3 & 0.93 & 65 & 21.67 \\
\hline Shephard, K & 3 & 0.93 & 50 & 16.67 \\
\hline Shin, J & 3 & 0.93 & 0 & 0 \\
\hline Vega-Marcote, P & 3 & 0.93 & 34 & 11.33 \\
\hline Sureda-Negre, J & 3 & 0.93 & 29 & 9.67 \\
\hline Álvarez, O & 2 & 0.62 & 18 & 9 \\
\hline
\end{tabular}

Si se presta atención a las universidades que más destacan en lo que a producción científica se refiere, se distinguen diferentes instituciones procedentes de diferentes lugares del mundo que publican sobre esta línea de investigación, lo que se ilustra en la tabla 5. Así, la Universidade Federal de Santa Catarina, University of Eastern Finland, la Universidad de Granada y University of PlyMouth encabezan la lista, con un total de cinco contribuciones. Atendiendo al índice de impacto, es la Universidade da Coruna aquella cuyos trabajos recogieron mayor índice de citación por cantidad de trabajo.

Tabla 5. Instituciones con mayor número de publicaciones.

\begin{tabular}{|l|c|c|c|c|}
\hline \multicolumn{1}{|c|}{ Instituciones } & № doc & $\%$ & Citas & Índice de impacto \\
\hline Universidade Federal de Santa Catarina & 5 & 1.553 & 12 & 2.40 \\
\hline University of Eastern Finland & 5 & 1.553 & 65 & 13 \\
\hline University of Granada & 5 & 1.553 & 38 & 7.60 \\
\hline University of PlyMouth & 5 & 1.553 & 48 & 9.60 \\
\hline Universidade da Coruna & 4 & 1.242 & 38 & 9.50 \\
\hline Universidade Federal de Santa Maria UFSM & 4 & 1.242 & 2 & 0.50 \\
\hline Universidade Federal de Sao Carlos & 4 & 1.242 & 9 & 2.25 \\
\hline Kazan Federal University & 3 & 0.932 & 0 & 0 \\
\hline Autonomous University of Madrid & 3 & 0.932 & 10 & 3.33 \\
\hline National Sun Yat Sen University & 3 & 0.932 & 8 & 2.66 \\
\hline
\end{tabular}


En último lugar, y con la finalidad de exponer las conexiones existentes entre los diferentes descriptores de los diversos documentos sobre la temática, se ha optado por la realización de un mapa bibliométrico con mapas de redes compuestos de nodos que evidencian las palabras clave de los documentos de la muestra tomada. El software empleado para esto ha sido VOSviewer, y ha creado un mapa de redes donde los nodos de mayor tamaño indican que esa palabra clave se emplea con más frecuencia que aquellas de menor tamaño. La figura 3 muestra por colores los diferentes grupos o clústeres que se interrelacionan entre sí formando colores intermedios. Así, destacan en primer lugar como aquellas palabras clave más empleadas "education", "health" y "higher education".

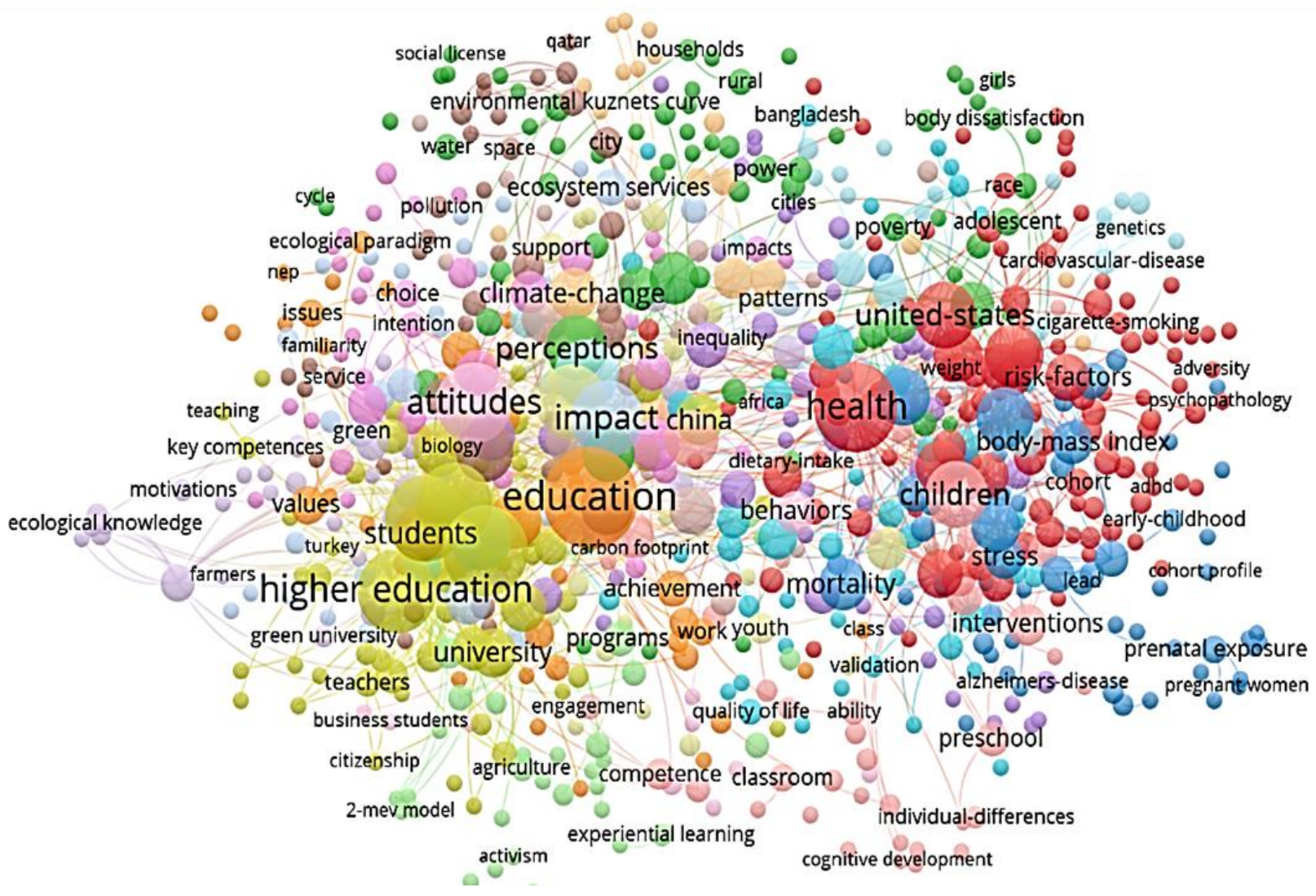

Fig. 3. Mapa de descriptores clave más utilizados en la muestra de artículos

\section{DISCUSIÓN}

El estudio emprendido permitió extraer metadatos de la producción científica de la Educación para el Desarrollo Sostenible en el contexto universitario. De esta forma, se identificaron un total de 322 artículos concentrados en el periodo comprendido entre 1970 y 2019. El aumento de la literatura científica relativa a esta disciplina y su impacto en la Educación Superior durante los últimos años, puede explicarse por el interés que ha suscitado entre la comunidad científica, como respuesta a la problemática ambiental envolvente y la responsabilidad a asumir desde la formación universitaria (Álvarez-Lires et al., 2017; De Matos et al., 2020).

En la comunidad científica se reconoce la importancia de avanzar en el desarrollo de estudios que permitan constatar la relevancia de educar para la sostenibilidad en la Educación Superior, teniendo en cuenta que el alumnado que se encuentra en formación en las aulas universitarias ocupará en el futuro puestos profesionales en diferentes escenarios laborales, constituyendo un motor de cambio imprescindible para responder a los nuevos desafíos sociales ante los que nos encontramos, entre los que figuran los referentes a la degradación medioambiental.

Entre los resultados más relevantes de esta investigación cabe destacar que la producción de trabajos sobre Educación para el Desarrollo Sostenible en el ámbito universitario se ha multiplicado exponencialmente en los últimos años, siendo su momento álgido en 2018, con 503 referencias científicas, hecho que concuerda con lo establecido en la ley bibliométrica de Price, así como con los resultados derivados de otras investigaciones, en las que se advierte que al encontrarnos en tiempos de incertidumbre social y crisis ecológica, los investigadores cuentan cada vez con mayores inquietudes para emprender estudios destinados a analizar el impacto de la crisis medioambiental en la Educación Superior, como forma de evidenciar la trascendencia de estas cuestiones en la formación de futuros profesionales (Antúnez y Gomera, 2017; Escámez y López, 2019; Espino-Román et al., 2015; Valderrama et al., 2020). 
Las temáticas más investigadas que se han apreciado en los estudios analizados se centran, fundamentalmente, en el marco de la salud ocupacional ambiental pública (22\%), las ciencias ambientales y la ecología (21\%) y en último lugar la investigación educativa (19\%). Con respecto a la primera de las temáticas, cabe destacar que los documentos que forman parte de esta investigación se han centrado en analizar los comportamientos de la población hacia el desarrollo sostenible, enfatizando la necesaria conjunción entre bienestar físico, psicológico y social, como factores necesarios para una relación más equilibrada y coherente con el entorno. Estos resultados coinciden con otros estudios (Mora-Mayoral y Martínez-Martínez, 2018; Murga-Menoyo, 2018), que identifican en la sostenibilidad un modo de impulsar valores de justicia, responsabilidad y tolerancia, como ejes centrales para promover un estilo de vida sostenible. Junto a ello, se enfatiza la necesidad de emprender un cambio profundo en las formas de vida y la organización social, de forma que la conciencia ambiental y la cultura ecológica puedan hacerse realidad.

En relación con los estudios enfocados en las ciencias ambientales y la ecología, ha de ponerse de manifiesto la relevancia otorgada a las actitudes, comportamientos y percepciones de estudiantes universitarios, considerando que estos deben ser ejes vertebradores de la educación actual. En este sentido, las investigaciones analizadas evidencian que el alumnado universitario reconoce la necesidad de abordar aspectos vinculados con la crisis ecológica a lo largo de la formación superior, en la medida en que la mejora del Planeta afecta a toda la humanidad, y, como tal, debe reflejarse en el futuro ejercicio profesional. Estos datos concuerdan con otros estudios (Álvarez-Lires et al., 2017; Cebrián et al., 2020; Espino-Román et al., 2015; Esteban et al., 2017), en los que se ha analizado la importancia que los estudiantes universitarios conceden al abordaje transversal de la Educación para el Desarrollo Sostenible, al tratarse de una herramienta pedagógica con la que concienciar acerca de la problemática ambiental actual.

En cuanto a los trabajos sobre investigación educativa, la mayor parte de las indagaciones ponen de manifiesto el compromiso que ha de asumir el profesorado en una educación sostenible, integrando cuestiones relacionadas con la degradación planetaria en el espacio docente de las asignaturas impartidas. En esta línea se sitúan otras investigaciones precedentes (Alcalá del Olmo, 2020; Alcalá del Olmo et al., 2020; Amaral et al., 2015; Sjögren, 2019) en las que se constata la necesidad de contar con un profesorado implicado, que ofrezca a los estudiantes todas aquellas experiencias de aprendizaje con las que desarrollarse personal y profesionalmente en el contexto de la sostenibilidad.

Además de apreciarse el carácter internacional de las publicaciones analizadas, cabe destacar, en relación con las revistas más productivas en las que figuran artículos relacionados con el eje central de este estudio, que gran parte de ellas están relacionadas con las áreas de la Educación Ambiental ("Environmental Education Research"), las repercusiones de la sostenibilidad en la Educación Superior ("International Journal of Sustainability in Higher Education") y la sostenibilidad en general ("Sustainability"). Por otra parte, las palabras clave de mayor tamaño extraídas de los documentos que han conformado la muestra de este trabajo, son higher education, health, attitudes, perceptions, education, entre otras. Ello indica que el principal objeto de estudio de esta temática se centra en cuestiones relacionadas con la salud, la ecología y las actitudes y percepciones ambientales en general, sin tener en cuenta tanto el impacto generado por el paradigma de la sostenibilidad en la Educación Superior.

Atendiendo a esta investigación bibliométrica, se advierte que el campo de indagación en Educación para el Desarrollo Sostenible en el contexto universitario es emergente, en la medida en que se reconoce la relevancia de esta disciplina como campo de conocimiento práctico y teórico. Las variables y disyuntivas económicas, políticas, sociales y culturales, que ayudan a identificar y entender la problemática ambiental, junto a la necesidad de concretar medidas que permitan afrontarla debidamente, hace percibir en esta disciplina una forma de trabajar valores vinculados con la sostenibilidad de forma sistemática y rigurosa en la universidad, convirtiéndose así en una propuesta pedagógica con la que desencadenar cambios actitudinales, cognitivos y relativos a las mentalidades.

Para hacer efectiva la implementación de la Educación para el Desarrollo Sostenible en las universidades, de la misma forma, resulta indispensable integrar la sostenibilidad entre sus objetivos estratégicos (Cebrián, et al., 2020), lo que implica la adopción de cambios que no solo inciden en la forma de concebir el currículum y los métodos tradicionales de enseñanza y aprendizaje, sino que lo trascienden de forma significativa, puesto que lo que se busca, principalmente, es integrar los Objetivos de Desarrollo Sostenible en el espacio docente de las diversas asignaturas, de tal forma que el estudiante llegue a ejercer su profesión desde la responsabilidad y la ética medioambiental. De la misma forma, es necesario prestar la debida atención a la formación del profesorado, incentivando el desarrollo de propuestas formativas que permitan, no solo tomar conciencia de la responsabilidad universitaria con la sostenibilidad, sino que ofrezcan orientaciones sobre la mejor forma de incluir cuestiones referentes a la degradación planetaria en el tejido curricular universitario.

Por otra parte, en el marco de la Extensión Universitaria, buscando articular espacios que permitan estrechar lazos entre universidad y comunidad, la Educación para el Desarrollo Sostenible debe trabajarse recurriendo 
a experiencias de aprendizaje cooperativo y de aprendizaje servicio (Antúnez y Gomera, 2017), al tratarse de metodologías que permiten hacer realidad una educación experiencial destinada a la mejora de la sociedad, además de propiciar un aprendizaje transformador y participativo, un pensamiento crítico y una total implicación de los estudiantes en su proceso de construcción de conocimientos. A todo ello ha de sumarse la importancia de que las universidades analicen en qué medida las metas de los Objetivos de Desarrollo Sostenible están presentes en aspectos relativos a la investigación, la innovación, la especialización y el asesoramiento, al objeto de emprender las oportunas acciones que permitan seguir avanzando en el camino hacia la sostenibilidad curricular.

\section{CONCLUSIONES}

A pesar de que los índices de productividad de la Educación para el Desarrollo Sostenible en el espacio universitario datan de varios años, la presencia de esta línea de investigación ha ganado relevancia en los últimos tiempos. Los diferentes Objetivos del Desarrollo Sostenible propuestos por las instituciones internacionales, reflejados en la Agenda 2030, han llevado a los expertos a indagar sobre esta temática, emergiendo así un número cada vez más creciente de investigaciones. Es por ello por lo que la producción científica en torno a esta línea de investigación ha crecido de forma significativa en los últimos años.

Este estudio presenta algunas limitaciones que deben ser tenidas en cuenta al interpretar los resultados. En este sentido, cabe destacar que, al analizar la producción científica indexada en WoS acerca de la temática tratada, se han encontrado documentos que abarcan desde el año 1970 al 2000, que no han resultado del todo significativos puesto que se alejan de la época actual. Es a partir del siglo XXI cuando la producción comienza a ser más significativa y vinculada con la realidad actual. Por otro lado, al revisar las áreas de publicación se han identificado algunas que pertenecían a la salud ocupacional ambiental pública, las ciencias ambientales y la ecología, alejadas del eje central de esta investigación.

\section{REFERENCIAS}

Alcalá del Olmo, M.J., Análisis de la implementación de la sostenibilidad en el escenario universitario portugués: Principales barreras y desafíos curriculares, Espacios, 41(16), 27-37 (2020)

Alcalá del Olmo, M.J., Santos-Villalba, M.J., Leiva, J.J., y Matas, A., Sostenibilidad curricular: una mirada desde las aportaciones del profesorado de la Universidad de Málaga, https://doi.org/10.15366/riejs2020.9.2.0, Revista Internacional de Educación para la Justicia Social, 9(2), 309-326 (2020)

Aleixo, A.M., Leal, S., y Azeiteiro, U., Conceptualization of sustainable higher education institutions, roles, barriers, and challenges for sustainability: an exploratory study in Portugal, https://dx.doi.org/10.1016/j.jclepro.2016.11.010, Journal of Cleaner Production, 172, 1664-1673 (2018)

Álvarez-Lires, M.A., Arias-Correa, A., Lorenzo-Rial, M.A., y Serrallé-Marzoa, F., Educación para la sustentabilidad: cambio global y acidificación oceánica, https://dx.doi.org/10.4067/S0718-50062017000200010, Formación Universitaria, 10(2), 89-102 (2017)

Amaral, L.P., Martins, N., y Gouveia, J.B., Quest for a sustainable university: a review, https://dx.doi.org/10.1108/IJSHE02-2013-0017, International Journal of Sustainability in Higher Education, 16(2), 155-172 (2015)

Antúnez, M., y Gomera, A., Sostenibilidad y currículum: Problemática y posibles soluciones en el contexto universitario español. Profesorado. Revista de currículum y formación del profesorado, 21(4), 197-214 (2017)

Barata, R., Castro, P., y Martins-Loução, M.A., How to promote conservations behaviors: the combined role of environmental education and commitment, https://dx.doi.org/10.1080/13504622.2016.1219317, Environmental Education Research, 23(9),1322-1334 (2017)

Bradford, S. C., Sources of information on specific subjects, http://dx.doi.org/10.1177/016555158501000406, Journal Information Science, 10(4), 173-180 (1934)

Cebrián, G., Junyent, M., y Mulá, I., Competencies in education for sustainable development: emerging teaching and research developments, https://dx.doi.org/10.3390/su1202059, Sustainability, 12(579), 1-9 (2020)

Cruz, M., Bibliometría y Ciencias Sociales, Clío: History and History Teaching, 7, 1-10 (1999)

De Matos, E., Leitão, J., y Alves, H., Skateholders' perceptions of sustainable development of higher education institutions: an intellectual capital approach, https://doi.org/10.1108/IJSHE-01-2020-0030, International Journal of Sustainability in Higher Education, 21(5), 911-942 (2020)

Diem, A., y Wolter, S., The use of bibliometrics to measure research performance in education sciences, https://dx.doi.org/10.1007/s11162-012-9264-5, Research in Higher Education, 54(1), 86-114 (2013)

Espino-Román, P., Olaguez-Torres, E., y Davizon-Castillo, Y.A., Análisis de la percepción del medio ambiente de los estudiantes de ingeniería en mecatrónica, https://dx.doi.org/10.4067/S0718-50062015000400006, Formación Universitaria, 8(4), 45-54 (2015) 
Esteban, M., Amador, L.V., y Mateos, F., Attitudes of university students towards the environment: environmental education and innovation, Revista de Humanidades, 31, 17-38 (2017)

Fernández-Cano, A., y Bueno-Sánchez, A., Síntesis de estudios bibliométricos españoles en educación. una dimensión evaluativa, Revista Española de Documentación Científica, 21(3), 269-285 (1998)

Geli de Ciurana, A.M., Collazo, L.M., y Mulá, I., Contexto y evolución de la sostenibilidad en el currículum de la universidad española. https://doi.org/10.25267/Rev_educ_ambient_sostenibilidad.2019.v1.i1.1102, Revista de Educación Ambiental y Sostenibilidad, 1 (1), 1102 (2019)

Gutiérrez-Braojos, C., Martín-Romera, A., y otros tres autores, Análisis temático de la investigación educativa soportada por la grounded theory, https://doi.org/10.13042/Bordon.2016.41035, Bordón, Revista de Pedagogía 69(1), 83-102 (2016)

Jorgenson, S.N., Estephens, J.C., y White, B., Environmental education in transition: a critical review of recent research on climate change and energy education, https://dx.doi.org/10.1080/00958964.2019.1604478, The Journal of Environmental Education, 50(3), 160-171 (2019)

Medina, I.F., y Páramo, P., La investigación en educación ambiental en América Latina: un análisis bibliométrico, Revista Colombiana de Educación, 66, 55-72 (2014)

Miranda, A., Bibliometría, Bibliotecas, 8(1), 1-11 (1990)

Mora-Mayoral, M.J., y Martínez-Martínez, F.R., Desarrollo local sostenible, responsabilidad social corporativa y emprendimiento social, http://dx.doi.org/10.19052/ed.4375, Equidad y Desarrollo, 31, 27-46 (2018)

Mulà, I., Tilbury, D., y otros siete autores, Catalyzing change for sustainability in higher education: a review of professional development initiatives for university educators, https://dx.doi.org/10.1108/lJSHE-03-2017-0043, International Journal of Sustainability in Higher Education, 18(5), 798-820 (2017)

Murga-Menoyo, M.A., La formación de la ciudadanía en el marco de la Agenda 2030 y la justicia ambiental, https://doi.org/10.15366/riejs2018.7.1.002, Revista Internacional de Educación para la Justicia Social, 7(1), 37-52 (2018)

ONU, Transformar nuestro mundo: la agenda 2030 para el desarrollo sostenible, http://unctad.org/meetings/es/SessionalDocuments/ares70d1_es.pdf (2015)

Price, D.J.S., Little Science, big science... and beyond, Columbia University Press, N.Y., (1986)

Purcell, W.M., Henriksen, H., y Spengler, J.D., Universities as the engine of transformational sustainability toward delivering the sustainable development goals: "living labs" for sustainability, https://doi.org/10.1108/lJSHE-02-2019-0103 International Journal of Sustainability in Higher Education, 20(8), 1343-1357 (2019)

Sjögren, H., More of the same: a critical analysis of the formations of teacher students through education for sustainable development, https://doi.org/10.1080/13504622.2019.1675595, Environmental Education Research, 25(11), 1620-1634 (2019)

UNESCO, Education for sustainable development goals. Learning objectives, https://unesdoc.unesco.org/ark:/48223/pf0000247444 (2017)

Valderrama, R., Alcántara, L., y otros seis autores, ¿Forma en sostenibilidad el sistema universitario español? visión del alumnado de cuatro universidades, https://dx.doi.org/10.5944/educXX1.23420, Educación XX1, 23(1), 221-245 (2020)

Wright, T., y Liang, Y., Examining the scholarly literature: a bibliometric study of journal articles related to sustainability and the arts, https://dx.doi.org/10.3390/su11143780, Sustainability, 11, 3780 (2019) 
